\title{
Biological Function of MicroRNA193a-3p in Health and Disease
}

\author{
Ilaria Grossi, Alessandro Salvi, Edoardo Abeni, Eleonora Marchina, and Giuseppina De Petro
}

Division of Biology and Genetics, Department of Molecular and Translational Medicine, University of Brescia, Brescia, Italy

Correspondence should be addressed to Alessandro Salvi; alessandro.salvi@unibs.it

Received 12 May 2017; Accepted 26 July 2017; Published 5 September 2017

Academic Editor: Michele Purrello

Copyright (c) 2017 Ilaria Grossi et al. This is an open access article distributed under the Creative Commons Attribution License, which permits unrestricted use, distribution, and reproduction in any medium, provided the original work is properly cited.

\begin{abstract}
MicroRNAs (miRNAs) are a class of small noncoding RNAs that act mainly as negative regulators of gene expression. Several studies demonstrated that miRNAs take part in numerous biological processes, such as proliferation, apoptosis, and migration. The dysregulation of miRNAs has been frequently observed in different types of disease, including cancer. Here, we provide a comprehensive review on the human miR-193a-3p by considering its role in both physiological and pathological contexts. Different mechanisms involved in regulating miR-193a-3p expression have been reported, including epigenetic modifications and transcription factors. In physiological contexts, miR-193a-3p seemed able to limit proliferation and cell cycle progression in normal cells. Remarkably, several publications demonstrated that miR-193a-3p acted as a tumor suppressor miRNA in cancer by targeting different genes involved in proliferation, apoptosis, migration, invasion, and metastasis. Furthermore, the downregulation of miR-193a-3p has been observed in many primary tumors and altered levels of circulating miR-193a-3p have been identified in serum or plasma of cancer patients and subjects affected by Parkinson's disease or by schizophrenia. In a clinical perspective, further studies are needed to explore the antitumor effects of the miR-193a-3p mimics delivery and the relevance of this miRNA detection as a possible diagnostic and prognostic biomarker.
\end{abstract}

\section{Introduction}

MicroRNAs (miRNAs) constitute a biologically very important class of small, noncoding RNAs, about 18-22 nucleotides (nt) long that mainly act as negative regulators of gene expression at posttranscriptional level by controlling the translation and stability of mRNA target. It is known that a miRNA may target several mRNAs as well as a mRNA can be under the control of several miRNAs. Most of the findings reported in the literature show clearly that miRNAs play an important role in several physiological and pathological processes exerting a highly precise regulation of most mRNA expression.

In this review, we focused on the human miR-193a-3p since the increasing number of evidences has described its importance in several biological functions. Moreover, the role as an important tumor suppressor miRNA has recently emerged in both liquid and solid tumors. According to UCSC Genome Browser (Human Dec. 2013 Assembly - GRCh38/ hg38) [1], miR-193a coding gene, defined as MIR193a, is located on human chromosome 17q11.2 (chr17:31,558,803$31,560,358$ ) (Figure 1(a)). By analyzing the region of $2000 \mathrm{bp}$ spanning MIR193a, a typical CpG island is identified (chr17:31558803-31560358) in which miR-193a coding sequence is embedded. Interestingly, MIR193a is found internal to a sequence that displays a high level of enrichment of $\mathrm{H} 3 \mathrm{~K} 27 \mathrm{Ac}, \mathrm{H} 3 \mathrm{~K} 4 \mathrm{Me} 1$, and $\mathrm{H} 3 \mathrm{~K} 4 \mathrm{Me} 3$ histone marks. In detail, the acetylation of lysine 27 of the $\mathrm{H} 3$ histone protein, the monomethylation of lysine 4 of the $\mathrm{H} 3$ histone protein, and the trimethylation of lysine 4 of the $\mathrm{H} 3$ histone protein have been associated with enhanced transcription, enhancer, and active promoter, respectively. In addition, a regulatory region characterized by transcription factor binding sites is found upstream MIR193a (chr17:31558470-31559544) indicating that miR-193a coding sequence is localized in an active transcriptional region. The pre-miR-193a generates two mature miRNAs, miR-193a-3p and miR-193a-5p, depending on the arm that is processed during miRNA biogenesis (Figure 1(b)). Consequentially, the different sequence that characterizes miR-193a-3p and miR-193a-5p determines distinct target sets for each miRNA. In this review, we focused on the miR-193a-3p biological and molecular mechanisms both from the physiological and the pathological perspectives. 


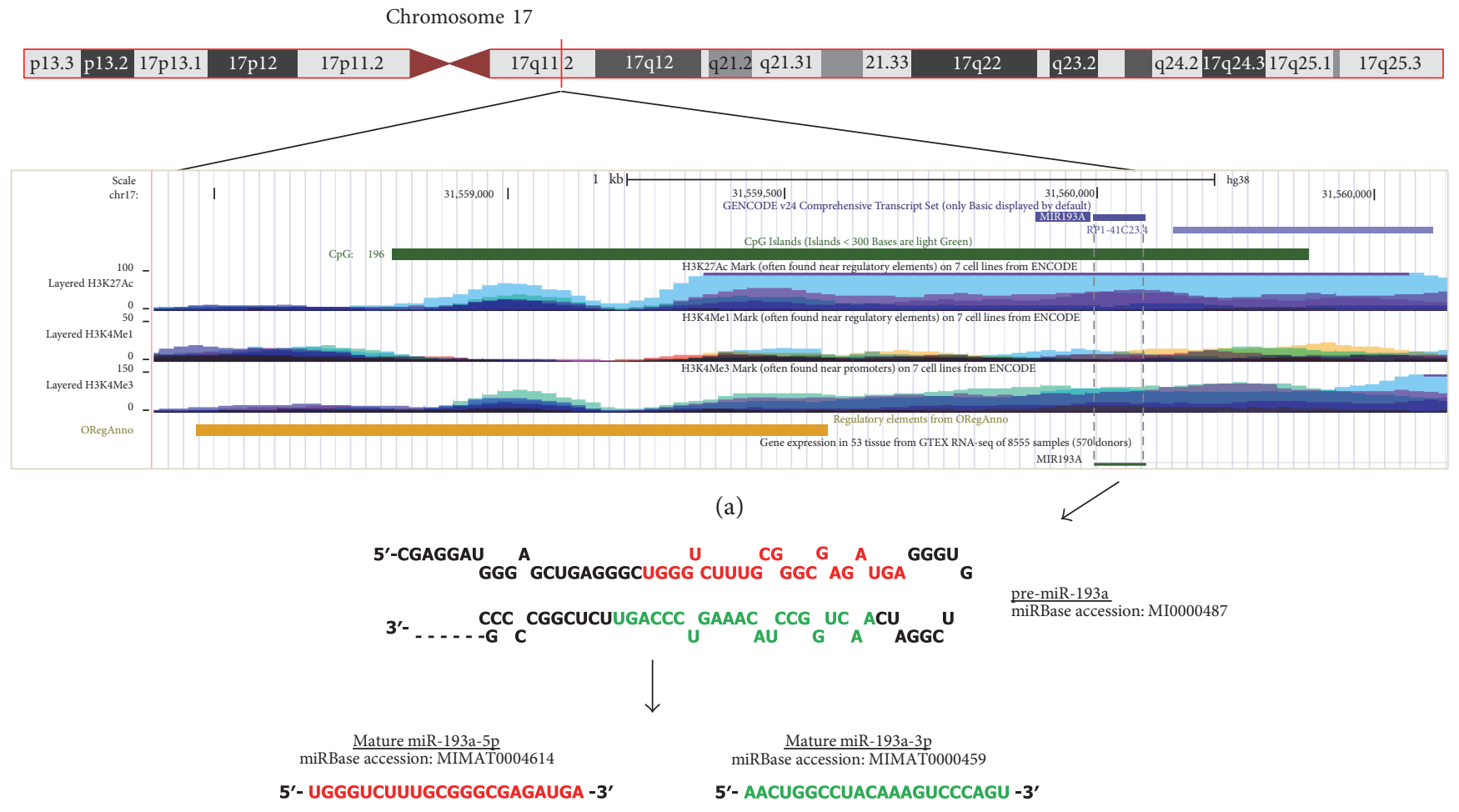

(b)

FIgURE 1: Genomic location of miR-193a coding sequence, stem-loop hairpin structure of pre-miR-193a and miR-193a-3p/miR-193a-5p sequences. (a) The analysis of the genomic region coding miR-193a referred to Genome Browser (https://genome-euro.ucsc.edu/). MIR193a coding sequence is located on human chromosome 17q11.2 characterized by a typical CpG island (in green). The layered $\mathrm{H} 3 \mathrm{~K} 27 \mathrm{Ac}, \mathrm{H} 3 \mathrm{~K} 4 \mathrm{Me1}$, and H3K4Me3 show the levels of histone marks across the genome in 7 cell lines (data obtain from ENCODE on the basis of ChiP-seq assay). By default, this track displays data from a number of cell lines in the same vertical space and each of the cell line is associated with a particular color. The regulatory element (in orange) is described as transcription factor binding sites by ORegAnno (open regulatory annotation). (b) The MIR193a gene is transcribed into a precursor (pre-miR-193a) with 88 nucleotides that is processed during miRNA biogenesis to yield mature miR-193a-5p (in red) and mature miR-193a-3p (in green) with 22 nucleotides in length.

Furthermore, the mechanisms involved in its expression regulation are also addressed. Finally, we highlighted the aberrant expression of miR-193a-3p both at tissue and at circulating levels in several pathological conditions, including cancer, in order to offer novel insights in the role of miR-193a-3p as a diagnostic and prognostic biomarker.

\section{The Regulation of miR-193a-3p Expression}

miR-193a-3p is highly conserved across several Hominidae (humans, chimpanzees, orangutans, and rhesus) and other mammals (Mus musculus, Bos taurus, and Canis familiars), as indicated in the microRNA viewer database (last update February 28, 2012) [2]. Several mechanisms, including transcription factors, DNA methylation, and competing endogenous RNAs (ceRNAs), have been reported to be involved in the dysregulation of $\mathrm{miR}-193 \mathrm{a}-3 \mathrm{p}$ in pathological contexts (Table 1). These evidences unmistakably suggest a multifactorial regulation of miR-193a-3p at transcriptional or posttranscriptional level with the possibility of a contextdependent activation of specific mechanisms.
2.1. Transcription Factors and Regulatory Proteins. Like protein-coding genes, the expression of miRNAs may be under the control of transcription factors (TFs) that bind to specific DNA sequences in the miR promoter and may act either as transcriptional activators or as repressors. The transcription silencing by specific TFs was reported to play a critical role in the inactivation of $\mathrm{miR}-193 \mathrm{a}-3 \mathrm{p}$ in different pathological contexts. Iliopoulos et al. showed that the downregulation of miR-193a-3p was driven by Max, the Myc-associated factor $\mathrm{X}$, and $\operatorname{RXR} \alpha$, a nuclear receptor, both involved in the processes causing cellular transformation of breast epithelial cells. Using chromatin immunoprecipitation (ChIP) and siRNA-mediated inhibition, they demonstrated that Max and RXR $\alpha$ bound directly to the miR-193a regulatory region and repressed its transcription in ER-Srctransformed cells [3]. Similarly, Li et al. revealed that the downregulation of miR-193a-3p was strongly associated with fusion protein AML1/ETO expressed in hematopoietic cells isolated from patients affected by acute myeloid leukemia (AML) with $\mathrm{t}(8 ; 21)$. In this pathological context, AML1/ ETO acted as a transcriptional repressor by localizing the AML1 binding site on the MIR193a upstream region and 
TABLE 1: Regulation of miR-193a-3p expression by different mechanisms.

\begin{tabular}{|c|c|c|c|c|}
\hline Mechanisms of regulation & $\begin{array}{l}\text { Effect on miR-193a-3p } \\
\text { expression }\end{array}$ & Sample type & Experimental procedures & Ref. \\
\hline \multicolumn{5}{|l|}{ Transcription factors: } \\
\hline Max and $\operatorname{RXR} \alpha$ & Downregulation & Transformed breast epithelial cells & $\begin{array}{l}\text { ChIP; siRNA-mediated } \\
\text { inhibition experiments }\end{array}$ & {$[3]$} \\
\hline AML1/ETO & Downregulation & $\begin{array}{l}\text { AML cell lines primary AML } \\
\text { samples with } t(8 ; 12)\end{array}$ & Luciferase reporter assay; ChIP & {$[4]$} \\
\hline $\mathrm{HNF} 4 \alpha$ & Upregulation & $\begin{array}{l}\text { Liver from mice with liver-specific } \\
\text { knockout of } \mathrm{HNF} 4 \alpha\end{array}$ & $\begin{array}{l}\text { miRNA microarray and qPCR } \\
\text { in Hnf } 4 \alpha \text {-LivKO mice }\end{array}$ & {$[5]$} \\
\hline \multicolumn{5}{|l|}{ DNA methylation: } \\
\hline DNA hypermethylation & Downregulation & $\begin{array}{c}\text { AML cell lines } \\
\text { AML primary samples }\end{array}$ & $\begin{array}{c}\text { MSP } \\
\text { Bisulfite sequencing }\end{array}$ & [9] \\
\hline DNA hypermethylation & Downregulation & $\begin{array}{l}\text { OSC carcinoma cells and primary } \\
\text { AML samples }\end{array}$ & $\begin{array}{c}\text { COBRA } \\
\text { Bisulfite sequencing }\end{array}$ & [8] \\
\hline DNA hypermethylation & Downregulation & $\begin{array}{l}\text { NSCL cancer cells } \\
\text { NSCL specimens }\end{array}$ & $\begin{array}{c}\text { MSP } \\
\text { Bisulfite sequencing }\end{array}$ & {$[10,11]$} \\
\hline DNA hypermethylation & Downregulation & Highly metastatic osteosarcoma cells & Bisulfite sequencing & {$[12]$} \\
\hline DNA hypomethylation & No effect & $\begin{array}{l}\text { HCC cell lines } \\
\text { HCC specimens }\end{array}$ & MSP & [14] \\
\hline DNA hypomethylation & No effect & Mesothelioma & MSP & [13] \\
\hline \multicolumn{5}{|l|}{ Regulatory protein: } \\
\hline XB130 & Downregulation & Thyroid carcinoma cells & $\begin{array}{l}\text { shNA-mediated inhibition and } \\
\text { ectopic expression experiments }\end{array}$ & [7] \\
\hline \multicolumn{5}{|l|}{$\begin{array}{l}\text { Competing endogenous RNA } \\
\text { (ceRNA) network: }\end{array}$} \\
\hline Linc00152 & Downregulation & Colon cancer cells & RIP; luciferase reporter assay & [17] \\
\hline LncRNA-UCA1 & Downregulation & NSCL cells & RIP; luciferase reporter assay & [18] \\
\hline
\end{tabular}

recruiting HDAC and DNMTs. The chromatin remodeling complex formed by AML1/ETO and the DNA hypermethylation triggered the silencing of miR-193a-3p in $t(8 ; 21)$ AML [4].

Other two factors, hepatocyte nuclear factor $\alpha(\mathrm{HNF} 4 \alpha)$ and XB130, may also have a relevant role in the regulation of miR-193a-3p. HNF $4 \alpha$ is a regulator of hepatic gene expression essential for liver development and function. The lacking of HNF $4 \alpha$ expression in the liver of young adult mice (Hnf $4 \alpha$-LivKO) determined the downregulation of some miRs, including miR-193a that is in cluster with miR-365 on the chromosome 11 of Mus musculus [5]. $\mathrm{XB130}$ is a member of the actin filament-associated protein (AFAP) family affecting the downstream signaling PI3k/Akt pathway by functioning as an adaptor protein and tyrosine kinase substrate [6]. In human thyroid carcinoma WRO cells and MRO cells, the gene silencing of XB130 by stable transfection of short hairpin increased both pri-miR-193a and its mature form (miR-193a-3p), while the ectopic expression of XB130 induced their downregulation [7]. These data indicated that the regulation of miR-193a-3p may be mediated by HNF $4 \alpha$ and XB130 in a healthy liver and thyroid carcinoma, respectively. However, further studies based on ChIP and gene reporter assays are needed in order to examine the direct and specific mechanisms that link these factors to $\mathrm{miR}$ expression.
2.2. Epigenetic Regulation by DNA Methylation. The hypermethylation of $\mathrm{CpG}$ islands located around miR genes is a key mechanism of epigenetic downmodulation of miRs that acts as a tumor suppressor in specific tumors. The MIR193A gene is embedded in a 1556 bp CpG island that counts 196 CpG sites (Figure 1(a)). Several studies have found that altered DNA methylation occurring in the CpG sites of the miR-193a promoter in different types of tumors. The miR$193 a-3 p$ was silenced in oral squamous cell carcinoma (OSCC) cell lines and in primary tumors through aberrant DNA methylation of the CpG sites near the miR coding sequence as verified by COBRA (combined bisulfite restriction analysis) assay and bisulfite sequencing [8]. Gao et al. demonstrated that the promoter hypermethylation repressed miR-193a-3p expression in acute myeloid leukemia (AML). The authors studied the DNA methylation levels in several leukemia cell lines and bone marrow (BM) samples from AML patients and healthy donors by bisulfite sequencing and methylation-specific PCR (MSP). Treatment with the inhibitor of DNA methylation 5-azacytidine (5-aza-dC) restored miR-193a-3p expression and reduced its target, the oncogene c-kit. In this situation, the growth inhibition and the induction of apoptosis and differentiation of AML cells were observed [9]. miR-193a was also found tumor specifically methylated in patients with non-small-cell lung cancer (NSCLC) [10]. Treatment with 5-aza-dC upregulated 
miR-193a-3p expression, impaired cell proliferation ability, and promoted apoptosis in NSCLC cells via downregulation of one of miR-193a-3p targets, the antiapoptotic myeloid leukemia cell sequence-1 (Mcl-1) [11]. Finally, Pu et al. found that both miR-193a-3p and miR-193a-5p were hypermethylated and downregulated in a metastatic osteosarcoma cell line [12].

Although altered DNA methylation levels have been associated with several types of tumor, this cannot be generalized. In malignant pleural mesothelioma (MPM), miR-193a-3p was inhibited when compared to normal pleura, but the DNA hypermethylation of miR-193a-associated CpG island was not responsible for the inhibition of miR-193a-3p in MPM cells as verified by MSP [13]. Interestingly, the results obtained in human hepatocellular carcinoma (HCC) by Grossi et al. were in line with those in MPM. The authors demonstrated that the downmodulation of miR-193a-3p in HCC was not mediated by DNA methylation in a cohort of 30 matched peritumoral and HCC tissues from bioptic samples. However, the miR-193a-3p CpG sites resulted methylated in the differentiated HepG2 cells and the treatment with 5 -aza-dC led to miR-193a-3p increasing as observed also by $\mathrm{Ma}$ and colleagues $[14,15]$. These results would point toward a variable miR-193a dependence on CpG DNA methylation in HCC. In conclusion, it has been proved that DNA hypermethylation of $\mathrm{CpG}$ island associated to MIR193A gene was responsible for miR-193a-3p downmodulation in certain types of cancer which in turn led to increased expression levels of miR-193a-3p targets involved in cell malignant behavior. In other types of cancer, DNA methylation may not contribute to the regulation of this miR.

2.3. The Competing Endogenous RNA (ceRNA). In the recent years, the important results obtained through the application of high-throughput RNA-seq have shed a light on the complex landscape of long noncoding RNAs (lncRNAs). These are RNA typically longer than 200 nucleotides without protein-coding potential. Several studies established the biological functions of lncRNAs comprising transcriptional and posttranscriptional regulation and chromatin modification. Furthermore, some lncRNAs have been characterized for their ability to regulate miRNA function by competing for miRNA binding and decreasing the negative effect of miRNAs on their targets. For this reason, these lncRNAs have been named as competing endogenous RNAs (ceRNAs) or miRNA sponges [16].

Consistently with the competing endogenous RNA role of IncRNAs, two different ceRNAs have been found to target miR-193a-3p where the physical association with mature miR-193a-3p has been demonstrated by RNA immunoprecipitation (RIP) and luciferase reporter assays. In particular, oncogenic Linc00152 (long intergenic noncoding RNA 152) and LncRNA-UCA1 (urothelial carcinoma-associated 1) competitively bind miR-193a-3p in colon cancer and NSCLC cell lines, respectively $[17,18]$. Interestingly, Linc00152 and UCA1 functioned as miRNA sponges and suppressed the endogenous effect of miR-193a-3p by silencing the miR target ERBB4. In addition, the overexpression of Linc00152 or UCA1 increased cell growth through modulation of
ERBB4 while this effect was attenuated by transfection of miR-193a-3p mimics in both cell lines.

These evidences strongly suggested that the ceRNA regulatory network should be considered as a mechanism involved in the dysregulation of miR-193a-3p.

\section{Expression Profile of miR-193a in Human Normal Tissues}

To provide complete information on the global expression profile of miR-193a-3p in normal tissues, we referred to data deposited in Genome Browser. The data have been reported as the median gene expression levels in 51 tissues and 2 cell lines, based on RNA-seq data obtained from the NIH Genotype-Tissue Expression (GTEx) project $[19,20]$. This release is based on data from 8555 tissue samples obtained from the postmortem of 570 adult individuals with no evidence of disease. As indicated in Figure 2, the expression of miR-193a (without discriminating between miR-193a-3p and miR-193a-5p) has been detected in all tissues, with the exception of the bladder, some brain components (hippocampus, nucleus accumbens, and spinal cord), and cervix (endocervix) where miR-193a expression was not found. Adipose and breast tissues displayed the highest miR-193a expression level.

\section{Biological Function of miR-193a-3p in Development and in Cell Physiology}

Very little is known on the biological function of miR-193a-3p in cell physiology. To the best of our knowledge, there have not been reports on the role of this $\mathrm{miR}$ in development. Concerning its role in cell physiology, the main available data were obtained from studies on the following: (a) cordblood and peripheral blood endothelial colony-forming cells (CB/PB-ECFC) derived from donations of healthy subjects; (b) from skeletal muscle specimens of control subjects (CTRL) with no sign of muscle pathology detectable by immunohistochemistry; and (c) from endometrial epithelial cells of healthy volunteer women aged 18 through 36 years old. In particular, it has been found that miR-193a-3p was one of the 25 miRNAs differentially regulated in CB-ECFC versus PB-ECFC [21]. It was highly expressed in the lessproliferative $\mathrm{PB}-\mathrm{ECFCs}$ where its inhibition using anti-miR molecules improved the in vitro proliferation, migration, and vascular tubule formation of these cells. Conversely, miR-193a-3p was expressed at low amount in the proliferative CB-ECFCs with its in vitro ectopic overexpression limiting the proliferation and the cell cycle progression of these cells and consequent reduction of their vascular tubule formation and cell migration. Altogether, the data obtained by Khoo et al., by using the miRnome studies, in silico miRNA target database analyses combined with proteome arrays and luciferase reporter assays in $\mathrm{miR}$ mimic-treated ECFCs, allowed to identify the negative regulatory role of miR-193a-3p in the vascular function of these cells and in the proliferation and migration abilities of these cells via directly targeting the HMGB1 expression (Table 2). Thus, this miR has a regulatory role in cell physiology of ECFCs 


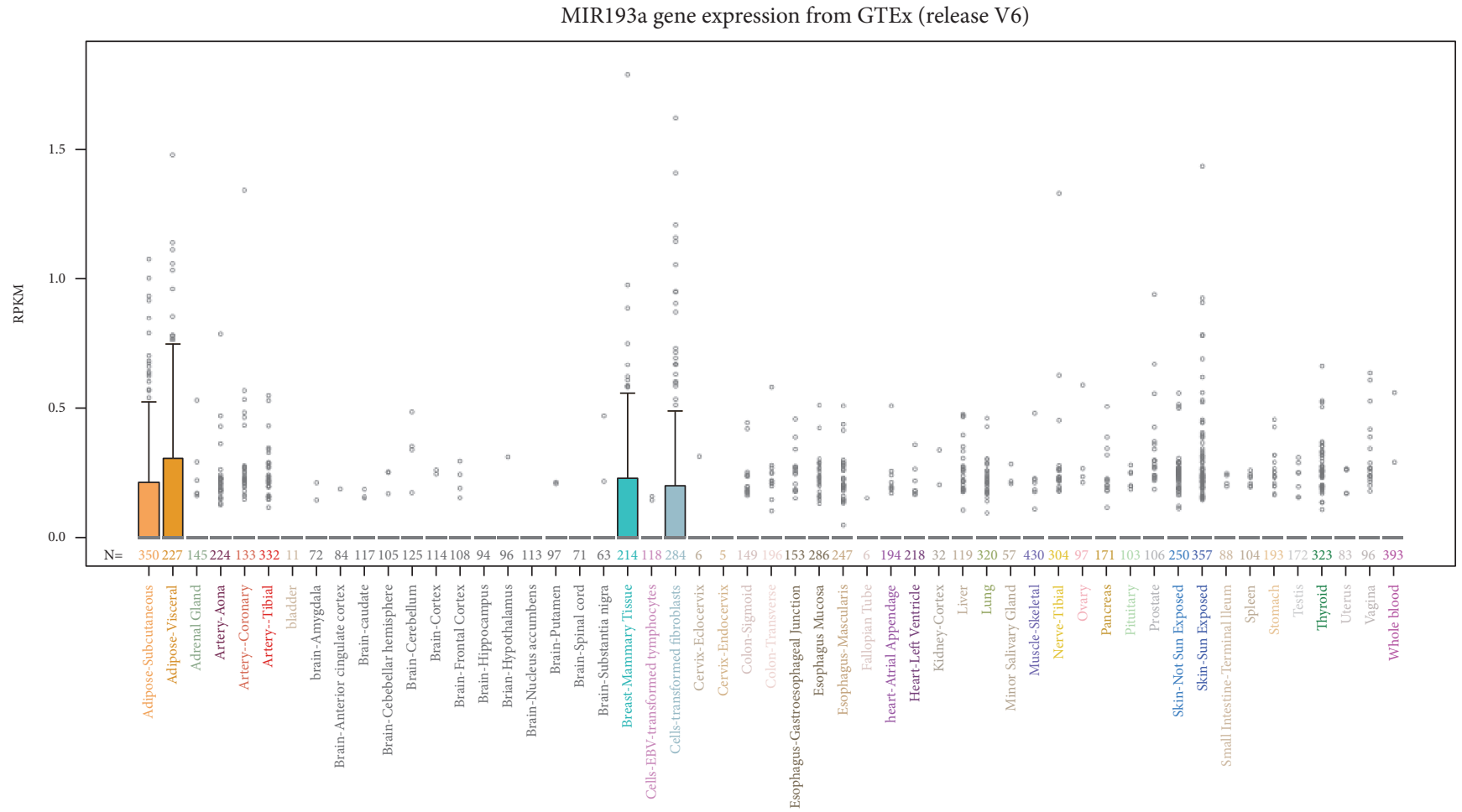

FIGURE 2: Expression profile of miR-193a in normal tissues. The expression level of miR-193a is reported for 51 tissues and 2 cell lines (EBV-transformed lymphocytes and transformed fibroblasts) and is referred to GTEx project collected in Genome Browser. Expression values are shown in RPKM (reads per kilobase of transcript per million mapped reads). The height of each bar represents the median expression level across all samples for a tissue, and points are outliers if they are above or below 1.5 times the interquartile range. Each color represents a specific tissue, conformed to GTEX consortium publication convention.

that are considered circulating endothelial lineage progenitors. Targeting the miR itself by anti-miR molecules may improve the abilities of PB-ECFC cells in proliferation and migration, in their angiogenic function thus contributing to a positive clinical outcome in ischemic diseases (stroke, myocardial infarction, and limb ischemia).

For skeletal muscle, a miRNA profiling approach combined with bioinformatics analyses and qPCR experimental validation has identified 11 miRNAs including miR-193a-3p involved in the homeostasis of normal myofibers. In particular, downregulation of miR-193a-3p has been associated with events contributing to the myofiber alterations of patients with myotonic dystrophy type 2 (DM2, OMIM 602688) [22]. DM2 is an autosomal dominant multisystemic disorder affecting the skeletal muscles, the heart, the eye, the central nervous system, and the endocrine system. The findings reported by Greco et al. clearly showed that the level of miR-193a-3p downmodulation contributed to the DM2 miRNA score allowing to distinguish the muscle specimens of DM2 patients from those of controls. However, these results do not permit to outline a hypothesis on the functional role in normal myofibers of miR-193a-3p as well as those of the other 10 miRNAs deregulated in DM2 muscle biopsies.

Finally regarding the human endometrium, several data suggested a regulatory function of miRNAs during the physiological cycle phases. In particular, Kuokkanen et al. provided strong evidence of the hormonal regulation of
miR-193a-3p expression in isolated uterine epithelial cells derived from midreproductive aged women [23]. These authors examined miRNAs at two stages: (a) in uterine epithelial cells derived from late proliferative phase biopsies (cycle day, $\mathrm{CD}, 12 \pm 1$ ) to target the time of maximal endometrial response to female steroid hormone estradiol-17 beta (E2) and (b) from secretory biopsies specimens from midluteal phase on CDs 19 through 23 to target the endometrial window of receptivity and maximum P4 (progesterone) action. The findings obtained using a genomic profiling of miRNAs and mRNAs clearly showed that miR-193a-3p is one of the 12 miRs found to be upregulated in the midsecretory phase samples. This expression is suggestive of a role in downregulating some cell cycle genes in the secretory phase thereby suppressing proliferation of the endometrial epithelial cells in this specific physiological context. Further, these data demonstrate hormonal regulation in miRNA (i.e., miR-193a-3p) expression in a human endometrium.

In summary, the overexpression of miR-193a-3p in cultured normal cells derived from physiological contexts, in particular in PB-ECFC cells and in midsecretory uterine epithelial cells, seemed to limit cell proliferation and cell cycle progression. However, the lack of data (KO, KI, and conditional KD) in the development of an organism (i.e., Mus musculus and Danio rerio) carrying the ortholog MIR193A (Gene Card data) has prevented the proposal of hypothesis on the role of miR-193a-3p during development [24]. 
TABLE 2: miR-193a-3p gene targets validated experimentally.

\begin{tabular}{|c|c|c|c|}
\hline Target genes & Functions & Cell line & References \\
\hline K-Ras & $\begin{array}{c}\text { Oncogene involved in many functions: antiapoptotic activity, } \\
\text { angiogenesis, motility, cell growth }\end{array}$ & $\begin{array}{l}\text { Breast cancer } \\
\text { Lung cancer }\end{array}$ & {$[3,29]$} \\
\hline PLAU & $\begin{array}{l}\text { Promotes cancer invasion and metastasis; the interaction of uPA } \\
\text { with its receptor induced also cell proliferation, migration, and } \\
\text { expression of specific genes }\end{array}$ & $\begin{array}{l}\text { Transformed breast epithelial cells } \\
\text { Breast cancer } \\
\text { HCC } \\
\text { BCa }\end{array}$ & {$[3,32,33,40]$} \\
\hline Mcl-1 & Antiapoptotic gene, member of the Bcl-2 family & $\begin{array}{l}\text { HeLa cells } \\
\text { Ovarian cancer }\end{array}$ & {$[30,31]$} \\
\hline ERBB4 & $\begin{array}{l}\text { Induces a variety of cellular responses including mitogenesis and } \\
\text { differentiation; triggers proliferation, invasion, and migration }\end{array}$ & $\begin{array}{l}\text { Lung cancer } \\
\text { NSCLC }\end{array}$ & {$[27,37]$} \\
\hline S6 K2 & Promote cell survival & NSCLC & {$[37]$} \\
\hline PepT1 & $\begin{array}{l}\text { Transporter involved in the low uptake of small bacterial peptides } \\
\text { in a normal colon and of dietary proteins }\end{array}$ & Epithelial colorectal adenocarcinoma & {$[46]$} \\
\hline c-kit & $\begin{array}{l}\text { Oncogene. Activates many pathways involved in proliferation, } \\
\text { differentiation, migration, and survival }\end{array}$ & AML & [9] \\
\hline Aml1/Eto & $\begin{array}{l}\text { Chimeric protein associated with the nuclear corepressor/histone } \\
\text { deacetylase complex to block hematopoietic differentiation }\end{array}$ & AML & {$[4]$} \\
\hline HDAC3 & Regulates transcription and modulates cell growth an apoptosis & AML & [4] \\
\hline DNMT3a & De novo DNA methylation & AML & [4] \\
\hline Cyclin D1 & Cell cycle progression & $\begin{array}{l}\text { AML } \\
\text { Breast cancer }\end{array}$ & $\begin{array}{c}{[4]} \\
{[28]}\end{array}$ \\
\hline E2F6 & Transcription factor with a main role in the control of the cell cycle & OSCC & {$[8]$} \\
\hline Rab27B & Increased invasion and metastasis in cancer & Osteosarcoma & [12] \\
\hline HMGB1 & $\begin{array}{l}\text { Tissue repair and regeneration, migration, angiogenesis, } \\
\text { endothelial recruitment, and proliferation }\end{array}$ & Endothelial colony forming cell, ECFC & [21] \\
\hline HYOU-1 & Cytoprotective role in hypoxia-induced cellular perturbation & Endothelial colony-forming cell, ECFC & [21] \\
\hline PSEN1 & Promote cell proliferation & $\begin{array}{l}\text { Esophageal squamous cell carcinoma } \\
\text { Bladder cancer }\end{array}$ & [45] \\
\hline $\mathrm{E} 2 \mathrm{~F} 1$ & Transcription factor; control of the cell cycle and apoptosis & $\mathrm{HCC}$ & [15] \\
\hline SRSF2 & $\begin{array}{l}\text { Regulates constitutive and alternative splicing; induces proapoptotic } \\
\text { splice forms of apoptotic genes }\end{array}$ & $\mathrm{HCC}$ & [15] \\
\hline HIC2 & Putative transcriptional factor & $\mathrm{BCa}$ & {$[40]$} \\
\hline HOXC9 & Transcription factor; role in morphogenesis & $\mathrm{BCa}$ & {$[43]$} \\
\hline ING5 & Inhibit cell growth and induce apoptosis & $\mathrm{BCa}$ & {$[42]$} \\
\hline LOXL4 & $\begin{array}{l}\text { Biogenesis of connective tissue: catalyzes the first step in the } \\
\text { formation of crosslinks in collagens and elastin }\end{array}$ & $\mathrm{BCa}$ & {$[41]$} \\
\hline SLC7A5 & Large neutral amino acid transporter small subunit 1 & Thyroid carcinoma & {$[7]$} \\
\hline JNK1 & $\begin{array}{l}\text { MAP kinases involved in proliferation, differentiation, } \\
\text { transcription regulation, and development }\end{array}$ & Breast cancer & [26] \\
\hline
\end{tabular}

\section{5. miR-193a-3p Functions as Tumor Suppressor miRNA in Cancer}

It is widely documented that the aberrant expression of miRNAs has a critical impact on cell biological processes and contributes to a number of pathological conditions, such as cancer. To the best of our knowledge, all published data pointed toward a role of miR-193a-3p as tumor suppressor miRNA (ts-miRNA) in both solid and liquid cancers since it impaired tumor cell aggressive properties by targeting oncogenes. In addition, miR-193a-3p is found downregulated in transformed cells and its downregulation seemed to be required for cellular transformation in two isogenic models (breast epithelial cells and fibroblasts) [3]. By considering a small cohort of cancer patients (only 36 cases), $\mathrm{Yi}$ et al. found that miR-193a-3p is upregulated in 24/36 esophageal squamous cell carcinoma (ESCC) tissues compared with adjacent normal tissues and the downregulation of miR-193a-3p by a synthesized inhibitor decreases migration and proliferation and promotes apoptosis in ESCC cells [25]. For these reasons, they described miR-193a-3p as an oncogenic miRNA in ESCC and suggested further studies to define the controversial role of miR-193a-3p in ESCC.

5.1. miR-193a-3p Limits Cancer Cell Proliferation and Impairs Cell Cycle Progression. Many studies have confirmed 
that miR-193a-3p has a significant role in the regulation of cancer cell growth. In particular, miR-193a-3p directly targeted JNK-1, a tyrosine kinase, since the ectopic expression of miR-193a-3p determined the dysregulation of cell cycle components including the decrease of CDK4, PIK3CA, and cyclin D1 and the overexpression of p27. In association to miR-124 and miR-147, miR-193a-3p has been shown to coregulate and inhibit G1/S transition and proliferation in breast cancer and glioblastoma cell lines [26]. Moreover, miR-193a-3p repressed cell proliferation of AML cells through the inhibition expression of c-kit, an oncogene encoding a transmembrane glycoprotein belonging to the type III receptor tyrosine kinase family [9]. In the same clinical context, miR-193a-3p has been also found to directly regulate the expression of DNMT3a, HDAC3, and cyclin D1 consequently blocking the cell cycle progression during granulopoiesis and inducing the differentiation of myeloid precursors [4]. It has also been shown that miR-193a-3p decreased the abilities of proliferation by the expression inhibition of some TFs, including E2F6, and other genes involved in the growth of several cancer types, for example, K-Ras, ERBB4, and cyclin D1 [3, 8, 27, 28]. In particular, it has been demonstrated that miR-193a-3p negatively regulated K-Ras in lung cancer cells by binding two $3^{\prime}$ UTR sites that have not been reported previously to be mutated in cancer. The overexpression of miR-193a-3p not only downregulated $\mathrm{K}$-Ras but also reverted the whole protein signature associated with the signaling downstream of K-Ras identified by proteomic analysis of lung cancer samples. The authors clearly determined the effects of miR-193a-3p on the cell aggressive properties via the targeting of K-Ras. Interestingly, miR-193a-3p decreased cell cycle progression (G1-S) and cell proliferation in vitro and blocked colony formation in threedimensional cultures. These findings have been translated into exciting ex vivo and in vivo experiments. For ex vivo experiments, the authors harvested lung-heart blocks from Sprague Dawley rats. To create a metastatic ex vivo 4D lung model, the lungs were decellularized and placed in a bioreactor with an oxygenator, pump with the right main stem ligated with silk suture, and A549 lung adenocarcinoma human epithelial cells were seeded in the left lung through the tracheal cannula. The use of 1,2-dioleoyl-sn-glycero-3phosphocholine (DOPC) nanoliposomes to deliver miR$193 a-3 p$ reduced the number of viable cells and impaired the presence of cancer cells in contralateral lobe that is indicative of metastasis formation in this model. In addition, this ex vivo model allowed the collection of circulating tumor cells (CTCs) from the perfused cell media present in a bioreactor bottle. Interestingly, cells derived from a $4 \mathrm{D}$ model treated with miR-193a-3p showed less proliferation ability than those from untreated model. In orthotopic xenograft K-Ras-mutated lung tumor models, miR-193a-3p encapsulated in DOPC nanoliposomes showed a reduction of tumor growth and metastasis at various sites [29].

5.2. miR-193a-3p Induces Cell Death Mainly by Promoting Apoptosis. When considering the miRNA target genes validated in different cancers, the role of miR-193a-3p in affecting genes with the consequence of promoting apoptosis stood out. In fact, among the putative targets, Mcl-1 is the most validated one involved in the programmed cell death. Mcl-1 is a multidomain protein belonging to the Bcl-2 family that binds and sequesters the $\mathrm{BH} 3$-only proapoptotic $\mathrm{Bcl}-2$ family members (Bim, Bid, Bik, Noxa, and Puma) which in turn induce Bak and Bax homo-oligomerization and activation. Kwon et al. demonstrated that miR-193a-3p expression was induced by ionizing irradiation in U-251 glioma cells and HeLa cells. miR-193a-3p negatively regulated Mcl-1 and promoted apoptosis by inducing ROS accumulation and DNA damage [30]. The direct binding of miR-193a-3p and Mcl-1 has also been demonstrated in human ovarian cancer cell line where the overexpression of miR-193a-3p induced the activation of caspase 3/7 and resulted in apoptotic cell death [31]. Furthermore, the transfection with miR-193a-3p mimics in MPM cells reduced Mcl-1 protein level and increased the number of late apoptotic cells. In addition, the release of lactate dehydrogenase (LDH) from MPM cells transfected with miR-193a-3p could suggest that miR-193a-3p induced cell death at least in part by the induction of necrosis. The ability of miR-193a-3p to promote apoptosis was further demonstrated in MPM xenograft models when targeted by miR mimics delivered using EDV nanocells, bacteriallyderived minicells that can be packaged with a variety of cargoes and be delivered to tumors via bispecific antibodies attached on surface [13]. Interestingly, Salvi et al. showed that the combination of miR-193a-3p mimics and sorafenib had additional effects on HCC inhibition of cell proliferation and induction of apoptosis suggesting that miR-193a-3p could also play an important role in promoting the sensitivity to sorafenib [32], the only innovative drug used for advanced HCC.

5.3. miR-193a-3p Impairs Cancer Migration, Invasion, and Metastasis. Tumor cell invasion and metastasis are events of primary importance in the prognosis of cancer patients. Metastatic cells are able to invade the basal membrane (BM) and extracellular matrix (ECM), to penetrate and move into the lymphatic or vascular circulation and to produce a secondary tumor by extravasation process and subsequent cell proliferation. Several evidences have highlighted the roles of miRNAs in these complex processes.

Recently, it has been demonstrated that miR-193a-3p acted as a negative regulator of urokinase-type plasminogen activator (UPA) in breast cancer and HCC cell lines and the high expression of miR-193a by mimics transfection strongly inhibited uPA expression and decreased cell aggressive properties $[3,32,33]$. uPA is a serine protease which converts the proenzyme plasminogen into the serine protease plasmin, thus making malignant cancer cells able to degrade $\mathrm{BM}$ and ECM. Furthermore, the interaction with its receptor, uPAR, leads to the activation of different intracellular signaling pathways, altering cell proliferation and migration abilities and expression of specific genes. The essential role played by uPA in migration has been well characterized in pathological context like cancer, and its overexpression was detected in various tumors, at both mRNA and protein level, representing an unfavorable prognostic factor [34-36]. 
Recently, Yu et al. validated miR-193a-3p as a negative regulator of ErbB4, belonging to the ErbB family of tyrosine kinase receptors and the ribosomal protein S6K2, both playing a critical role in cell movement, growth, and development [37]. The expression of miR-193a-3p and miR-193a-5p was positively associated with cellular invasion and migration by assessing human lung cancer cell with high metastatic potential (SPC-A-1sci) previously established from weakly metastatic cell (SPC-A-1) through in vivo selection in NOD/SCID mice [38]. Furthermore, the overexpression of miR-193a-3p inhibited migration, invasion, and epithelial mesenchymal transition in vitro and impaired the formation of metastasis in vivo. In addition, the protein profile of SPC-A-1sci cells stably transfected with miR-193a-3p has been determined by using a proteomic approach (iTRAQ and Nano LC-MS/MS) followed by DAVID (database for annotation, visualization, and integrated discovery; http:// david.abcc.ncifcrf.gov) and STRING analysis. Interestingly, 112 proteins resulted differentially expressed (62 upregulated and 50 downregulated) compared with miR controltransfected cells, and some of them have been associated to lung cancer metastasis and proliferation [39].

Similarly, $\mathrm{Pu}$ et al. reported that miR-193a-3p and miR-193a-5p were downregulated in osteosarcoma cells defined as highly tumorigenic and metastatic (MG63.2) in respect to less metastatic parental MG63 cell line. The abilities of MG63.2 cells to invade and migrate resulted decreased by restoring the miR-193a-3p expression level using transient transfection of miR mimics. Correspondingly, the inhibition of miR-193a-3p by antagomiR transfection in MG63 cells induced invasive properties. Furthermore, the authors identified Rab27, a member of the RabGTPase family, as a direct target of miR-193a-3p. By Cignal reporter finder assay, they showed that the Rab27 knockdown repressed some pathways that were clearly implicated in metastasis, including TGF $\beta$, Myc/Max, and ATF2/ATF3/ATF4. These results indicated the negative impact of miR-193a-3p on cancer invasion by repressing Rab27B and its downstream pathways in osteosarcoma cells [12].

Taken together, these evidences strongly suggest a potential role of miR-193a-3p as a metastasis-preventing miRNA. However, further work will be required to explore the exact molecular pathways by which this miRNA could exert its functions.

5.4. miR-193a-3p Modulates Drug Resistance in Cancer Cells. To date, chemotherapy still represents one of the most used therapeutic options for the treatment of solid tumors worldwide. However, the clinical efficacy of these treatments is limited by the onset of drug resistance and the side-effects of the drug both contributing to reduce cancer patients' positive outcome. Even if the specific regulatory mechanism involved in chemoresistance remains very often unclear, increasing evidences showed that miRNAs can have a crucial role in chemosensitivity by regulating cancer-related genes. As indicated in Figure 3(a), miR-193a-3p seems to be implicated in the activation of drug resistance pathways via repressing different targets. By a systematic analysis that compared the $\mathrm{H}-\mathrm{bc}$ multidrug resistant bladder cancer
(BCa) cells versus 5637 sensitive ones, Lv et al. reported that miR-193a-3p was silenced by DNA hypermethylation in the sensitive cells, the cell line presenting the lowest $\mathrm{IC}_{50}$ to different drugs. In chemoresistant BCa cells, miR-193a-3p decreased the expression of the following targets: SRSF2, PLAU, HIC2 [40], LOXL4 [41], ING-5 [42], HOXC9 [43], and PSEN-1 [44]. The last target has been considerate also relevant in chemo- and radioresistant esophageal cancer cell [45]. The results obtained through pathway reporter system assays revealed that the reduced level of these targets affected the activities of five signaling pathways in resistant cells. In particular, DNA damage, NF- $\kappa \mathrm{B}$, and Myc/Max pathways were found with lower activities, while Notch and oxidative stress pathways resulted activated in resistant cell compared with sensitive cells. In addition, the modulation of miR-193a-3p level by the injection of antagomir or agomir molecules in either resistant or sensitive BCa cells reversed the chemoresistance in tumor xenografts nude mice. Similarly, miR-193a-3p expression was found increased by DNA hypomethylation in $\mathrm{HCC}$ cells presenting resistance to 5-fluorouracil (5-Fu). miR-193a-3p seemed to induce antiapoptotic signals in 5 -Fu resistant cells by suppressing SRSF2, a splicing factor that preferentially upregulates the proapoptotic form of caspase 2 (CASP2L) to the antiapoptotic form CASP2S [15]. These intriguing data will require further investigations since it is not clear how the overexpression of miR-193a-3p can dictate chemoresistance even if its tumor suppressor functions have been well established in many primary cancers. In support of this notion, Yue et al. reported that the reduced activity of miR-193a-3p caused by the sponge effect of Linc00152 was related to oxaliplatin (L-OHP) resistance in colon cancer both in vitro and in vivo (Figure 3(b)). Linc00152 is usually overexpressed in human colon cancer tissues and is associated with poor prognosis in patients undergoing L-OHP treatment after surgery. Interestingly, Linc00152 competitively bound miR-193a-3p inducing the upregulation of its ERBB4 target and the consequent activation of AKT signaling pathway which, in turn, conferred resistance [17].

\section{Gene Annotation Analysis on Predicted and Experimentally Validated miR-193a-3p Targets}

It is well known that a given miR can regulate the expression of hundreds of targets, and conversely, dozens of miRs can target a single mRNA. For hsa-miR-193a-3p, TargetScan 7.1 predicted 293 putative target genes with 168 displaying the highest score (cumulative weighted context++ score $<-0.24)$. The functional annotation analysis conducted using DAVID 6.8 on the candidate target genes of miR-193a-3p highlighted 8 biological KEGG pathways overrepresented as statistically significant $(p<0.05)$. They were the following: microRNA in cancer, ErbB signaling pathway, GnRH signaling pathway, acute myeloid leukemia, PI3K-Akt signaling pathway, Ras signaling pathway, pathways in cancer, and focal adhesion. The importance of these pathways is indicated by the fact that they are highly relevant 


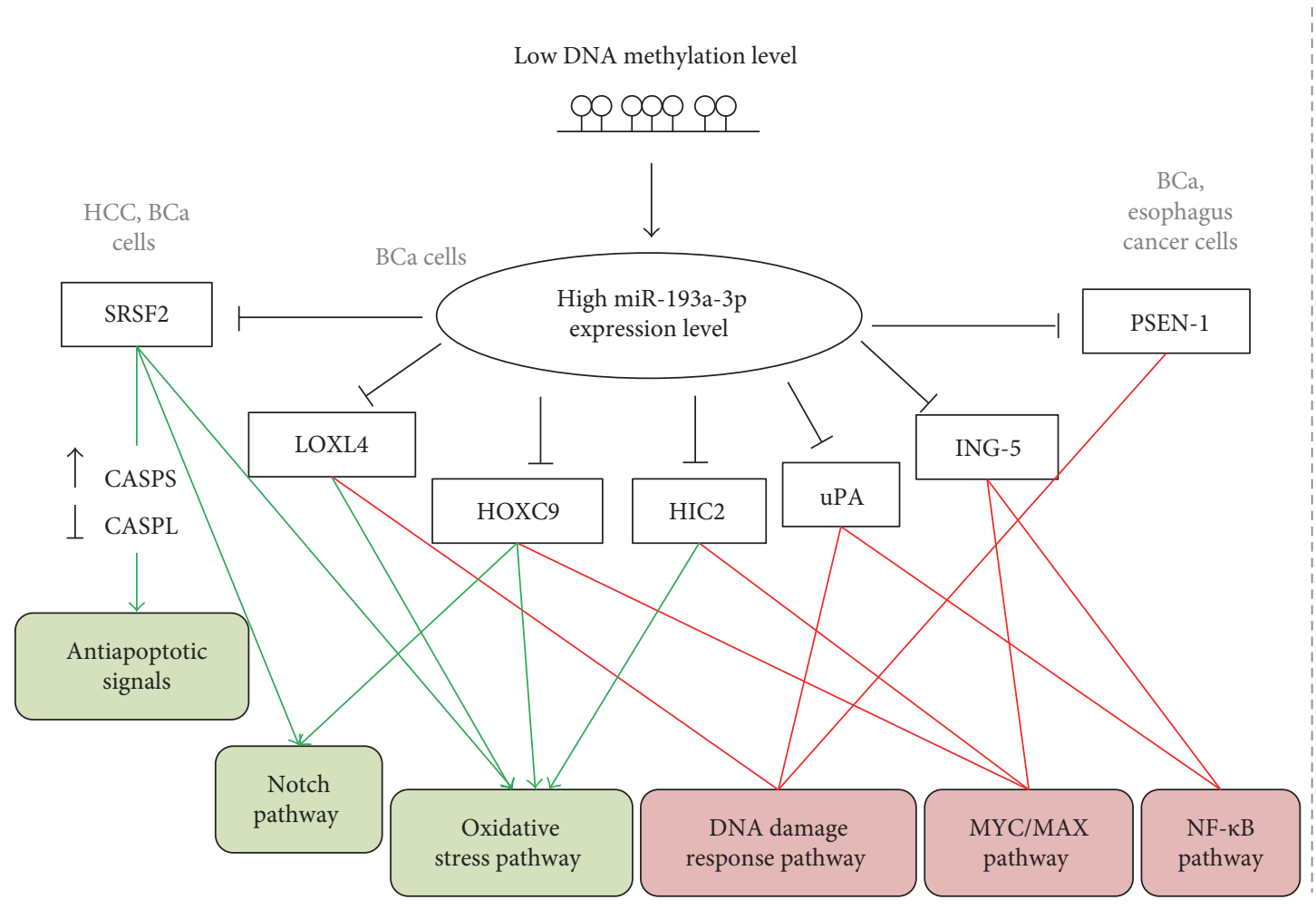

(a)
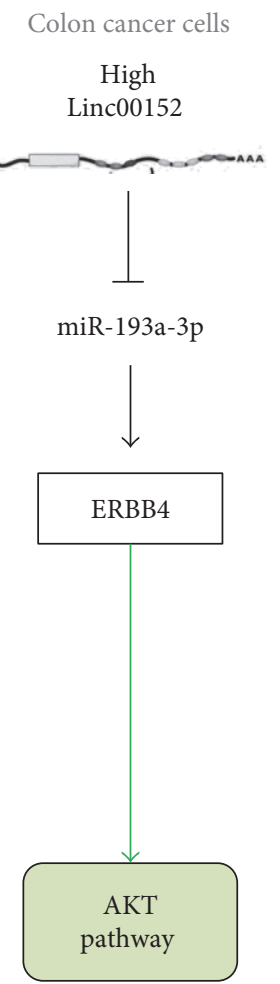

(b)

FIGURE 3: Proposed model for the role of miR-193a-3p in the regulation of the chemoresistance in different cancer cell lines. (a) Involvement of high miR-193a-3p expression level in chemoresistance in HCC cells, BCa cells, and esophageal cancer cells. (b) Low levels of miR-193a-3p are involved in resistance to oxaliplatin in colon cancer cells. Altered expression of miR-193a-3p will adversely affect immediate targets indicated inside black boxes. In turn, these targets will affect several downstream pathways with green arrows and red lines representing functional activation and repression, respectively.

in the processes of onset, progression, and metastasis of several types of cancer. In addition, among the 24 experimentally validated and published miR-193a-3p targets (Table 2), the KEGG pathway enrichment analysis outlines 6 terms $(p<0.05)$ : acute myeloid leukemia, chronic myeloid leukemia, microRNA in cancer, proteoglycans in cancer, Erb signaling pathway, pancreatic cancer, and pathways in cancer. This bioinformatics analysis underscored once again the key role of miR-193a-3p in cancer contexts.

\section{7. miR-193a-3p as Diagnostic and Prognostic Biomarker}

It is well known that the aberrant miRNA expression has a critical impact on many cell biological processes and contributes to a number of pathological conditions. For this reason, the study of miRNA expression profile in pathological contexts is necessary to support the clinical significance of a specific miRNA and its possible role as a diagnostic and prognostic biomarker.

7.1. Dysregulation of miR-193a-3p in Cancer Tissues. Consistent with the role of ts-miRNA, miR-193a-3p was found downregulated in the majority of primary cancer tissues, such as HCC [14, 47], NSCLC [37, 48], MPM [13], and AML [4, 9].
Furthermore, the low expression of miR-193a-3p was significantly related to reduced overall survival (OS) and disease-free survival (DFS) of HCC patients indicating its possible prognostic role in this cancer type [14]. In NSCLC, the expression of miR-193a-3p was negatively correlated to tumor size, lymph node metastasis (LNM), and TNM stages. Interestingly, miR-193a-3p was reported as downregulated in BRAF mutation with respect to wild-type melanoma [49] suggesting that miR-193a-3p may have a role in BRAFassociated events.

7.2. Circulating miR-193a-3p Levels in Pathological Conditions. Several data have assessed that circulating microRNAs in human body fluids (i.e., serum and plasma) offer unique opportunities as biomarkers for early diagnosis of clinical conditions [50]. Indeed, some small ncRNAs have been found highly stable under extreme conditions (i.e., extreme $\mathrm{pH}$ and temperature and ribonuclease digestion) and numerous studies of circulating microRNA profiling have been conducted for several diseases. In regard to circulating miR-193a-3p, the use of next-generation sequencing and $\mathrm{qPCR}$ revealed different levels of this $\mathrm{miR}$ in patients with schizophrenia or with Parkinson's disease (PD) when compared with control subjects. Regarding PD, serum miRNA level obtained from a small number of patients 
revealed that miR-193a-3p was among the panel of four miRNAs significantly decreased in the PD patients compared to controls. Furthermore, miR-193a-3p could also be used to distinguish the HY-stage1 in PD patients from healthy controls [51]. In schizophrenia, the higher level of plasma miR-193a-3p (and miR-130b) in patients compared to controls was determined by the global plasma miRNAs profiling in a test cohort of 164 schizophrenia patients and 187 control subjects and subsequently validated by qRT-PCR in an independent cohort of 400 schizophrenia patients [52]. We think that these findings are extremely interesting, but further studies are needed in order to support the detection of circulating miR-193a-3p as a noninvasive biomarker for these diseases.

Regarding cancer, circulating miR-193a-3p levels were found increased in many malignancies. By comparing two independent miRNA microarrays, one in tissue and one in blood of colorectal cancer patients, Yong et al. identified higher levels of miR-193a-3p (in combination to miR-23a and miR-338-5p) in cancer patients, and the positive correlation was demonstrated between tissue and blood samples [53]. The high-throughput TaqMan low-density array (TLDA) combined with qPCR validation allowed to establish the high level of miR-193a-3p included in two different fiveserum miRNA panels, either in renal cell carcinoma (RCC) or in NSCLC patients. It was clearly demonstrated by ROC analysis that the 5-miRNA-based panels (miR-193a-3p, miR-362, miR-572, miR-425-5p, and miR-543) had a high sensitivity and specificity in the discrimination of patients with early-stage RCC from healthy controls [54]. In NSCLC, the effectiveness of the 5-miRNA panel (miR-193a-3p, miR-483-5p, miR-214, miR-25, and miR-7) in discriminating cancer patients from normal subjects was confirmed in a multiethnic and multicentric study in which 438 participants from both China and America were enrolled (221 NSCLC patients, 161 normal controls, and 56 benign nodules) [55]. By using the same experimental approaches (TLDA followed by qPCR), Wu et al. identified significantly elevated levels of miR-193a-3p in sera from patients with esophageal squamous cell carcinoma (ESCC). The authors indicated that miR-193a-3p may be used to discriminate between ESCC cases and healthy controls with high sensitivity and specificity in a cohort of 63 patients and 63 controls. The level of circulating miR-193a-3p was reduced after ESCC surgical removal indicating that this miR may have been originally secreted by the tumor cells [56]. Interestingly, in a retrospective longitudinal phase 3 biomarker study, a set of 5 serum miRNAs (miR-193a-3p, miR-369-5p, miR-672, miR-429, and let-7i*) was identified as a specific biomarker for the surveillance and preclinical screening of HCC in a high-risk population of patients infected by hepatitis B virus (HBV). In particular, the different expression levels of these miRNAs (including the downregulation of miR-193a-3p) were identified in HBV patients who developed HCC (preclinical HCC patients) compared to the HBV group that did not develop HCC [57].

Although the origin of circulating miRNAs remains unclear, it has been reported that they may originate through different pathways including passive leakage from broken cells, active secretion via microvesicles, and active secretion through an RNA-binding protein-dependent pathway that has been suggested as the major source of circulating miRNAs $[58,59]$.

Extracellular vesicles (EVs) are mediators of intercellular communications during several physiopathological processes such as differentiation, tissue repair, proliferation, and apoptosis, and they are released from both cancer cells and noncancer cells. Among the EVs, exosomes are small vesicles $(50-150 \mathrm{~nm})$ able to transport and deliver proteins, mRNAs, and ncRNAs including miRs from a donor to recipient cells [60]. Oh et al. demonstrated that the exosomes containing miR-193a-3p were able to induce differentiation of F11 cells (rat dorsal root ganglion and mouse neuroblastoma hybrid cells). By a microfluidic assay that collected real-time images of exosomes migration, they verified that miR-193a-3p was a neurogenic miR that promoted the differentiation of recipient undifferentiated cells [61]. Teng et al. demonstrated that miR-193a-3p was present into exosomes obtained from tissue and cell culture media and serum, derived from primary mouse colon tumors and human liver metastasis of colon cancer. In severe disease, the high level of miR-193a-3p into the exosomes led to the reduction of cytoplasmatic miR-193a-3p that in turn promoted the progression of premetastatic cells to metastatic ones. The authors found that overexpression of MVP (major vault protein) transported miR-193a-3p from the tumor cells to exosomes. On the contrary, MVP knockout determined miR-193a-3p accumulation in tumor cells triggering the inhibition of cell proliferation and cell cycle G1 arrest due to miR-193a-3p binding to its target, caprin-1 [62].

\section{Conclusions and Perspectives}

In the last decade, an increasing number of evidences have proved the biological importance of miRNAs in physiological contexts and a huge number of studies have pointed to the fact that dysregulation of miRNAs plays a fundamental role in several pathological conditions, including cancer. In the present report, we focused on miR-193a-3p because several findings support its role as tumor suppressor miR both in solid and in liquid tumors leading to believe that the detection of this miR at tissue and/or circulating level may be employed as a diagnostic and prognostic biomarker for certain types of tumors. It is worth to outline that the functional role of a given miR can be tissue- or tumor-type dependent and that few data indicate a possible oncogenic role of miR-193a-3p in ESCC; nevertheless, we think that only strong clinical evidence, as well as biological studies of the miR mimics on the proliferation of ESCC cell lines will elucidate this possible role of miR-193a-3p in this specific context of human cancer.

From a general point of view, miR-193a-3p had been studied in Homo sapiens and, to the best of our knowledge, no information is available in development. Regarding the biological function in physiological contexts, certain types of human normal cells seem to require high expression level of miR-193a-3p when they do not need to proliferate. As a consequence, the cancer cells that usually need high proliferative capacity show low expression levels of this miR. 
Different factors can contribute to the regulation of MIR193a expression, including TFs, DNA methylation and, at posttranscriptional level, ceRNAs. The alteration of these factors is context dependent and determines the aberrant expression of miR-193a-3p in cancer. The elucidation of these mechanisms may allow extending our knowledge on the level of miR-193a-3p dysregulation.

As already mentioned, the dysregulation of a given microRNA may alter the expression of hundreds of genes in cancer affecting the entire network in which targets are involved. By considering all experimental validation studies in cancer, genes targeted by miR-193a-3p are involved in several biological processes, including proliferation, apoptosis, migration, and metastasis. To acquire major advancements in knowledge and comprehension of the canonical and noncanonical mRNA targets, more studies involving the use of proteomics profiling and RNA pull down with biotinylated microRNA mimics are needed.

To date, different clinical trials have demonstrated the use of miRNA-based therapy as a promising strategy for the treatment of different diseases, making miRNA highly relevant for a clinical use [63]. In this regard, the delivery of miR-193a-3p mimics by nanosized particles could represent a novel therapeutic tool for the treatment of cancer since it may hamper tumor aggressive properties in tumor xenograft models by restoring the miR original levels. On the other hand, the local delivery of anti-miR-193a-3p molecules could be an effective intervention for local ischemic diseases. These findings may pave the way to further studies aimed to elucidate the possible use of miR-193a-3p for experimental therapeutic procedures.

Finally, compelling evidences indicated that miR-193a-3p is detectable not only in primary cancer tissues but also at circulating level (in exosomes or not) in cancer patients indicating the possible diagnostic and prognostic value of miR-193a-3p. In addition, altered circulating levels of this miR have been identified in subjects affected by Parkinson's disease or schizophrenia. Further studies are really necessary to verify whether the detection of miR-193a-3p may be helpful for the characterization of these two diseases.

\section{Conflicts of Interest}

The authors declare that there is no conflict of interests regarding the publication of this paper.

\section{Acknowledgments}

The authors are grateful to Dr. M. Crosatti (University of Leicester, UK) for the linguistic revision of the manuscript.

\section{References}

[1] W. J. Kent, C. W. Sugnet, T. S. Furey et al., "The human genome browser at UCSC," Genome Research, vol. 12, no. 6, pp. 996-1006, 2002.

[2] A. Kiezun, S. Artzi, S. Modai, N. Volk, O. Isakov, and N. Shomron, "miRviewer: a multispecies microRNA homologous viewer," BMC Research Notes, vol. 5, p. 92, 2012.
[3] D. Iliopoulos, A. Rotem, and K. Struhl, "Inhibition of miR193a expression by Max and RXR $\alpha$ activates K-Ras and PLAU to mediate distinct aspects of cellular transformation," Cancer Research, vol. 71, no. 15, pp. 5144-5153, 2011.

[4] Y. Li, L. Gao, X. Luo et al., "Epigenetic silencing of microRNA193a contributes to leukemogenesis in $\mathrm{t}(8 ; 21)$ acute myeloid leukemia by activating the PTEN/PI3K signal pathway," Blood, vol. 121, no. 3, pp. 499-509, 2013.

[5] H. Lu, X. Lei, J. Liu, and C. Klaassen, "Regulation of hepatic microRNA expression by hepatocyte nuclear factor 4 alpha," World Journal of Hepatology, vol. 9, no. 4, pp. 191-208, 2017.

[6] R. Zhang, J. Zhang, Q. Wu, F. Meng, and C. Liu, "XB130: a novel adaptor protein in cancer signal transduction," Biomedical Reports, vol. 4, no. 3, pp. 300-306, 2016.

[7] H. Takeshita, A. Shiozaki, X. H. Bai et al., "XB130, a new adaptor protein, regulates expression of tumor suppressive microRNAs in cancer cells," PLoS One, vol. 8, no. 3, article e59057, 2013

[8] K. Kozaki, I. Imoto, S. Mogi, K. Omura, and J. Inazawa, "Exploration of tumor-suppressive microRNAs silenced by DNA hypermethylation in oral cancer," Cancer Research, vol. 68, no. 7, pp. 2094-2105, 2008.

[9] X. N. Gao, J. Lin, Y. H. Li et al., "MicroRNA-193a represses c-kit expression and functions as a methylation-silenced tumor suppressor in acute myeloid leukemia," Oncogene, vol. 30, no. 31, pp. 3416-3428, 2011.

[10] G. Heller, M. Weinzierl, C. Noll et al., "Genome-wide miRNA expression profiling identifies miR-9-3 and miR-193a as targets for DNA methylation in non-small cell lung cancers," Clinical Cancer Research, vol. 18, no. 6, pp. 1619-1629, 2012.

[11] J. Wang, B. Yang, L. Han et al., "Demethylation of miR-9-3 and miR-193a genes suppresses proliferation and promotes apoptosis in non-small cell lung cancer cell lines," Cellular Physiology and Biochemistry, vol. 32, no. 6, pp. 1707-1719, 2013.

[12] Y.Pu, F. Zhao, W. Cai, X. Meng, Y. Li, and S. Cai, "MiR-193a-3p and miR-193a-5p suppress the metastasis of human osteosarcoma cells by down-regulating Rab27B and SRR, respectively," Clinical \& Experimental Metastasis, vol. 33, no. 4, pp. 359-372, 2016.

[13] M. Williams, M. B. Kirschner, Y. Y. Cheng et al., "miR-193a-3p is a potential tumor suppressor in malignant pleural mesothelioma," Oncotarget, vol. 6, no. 27, pp. 23480-23495, 2015.

[14] I. Grossi, B. Arici, N. Portolani, G. De Petro, and A. Salvi, "Clinical and biological significance of miR-23b and miR193a in human hepatocellular carcinoma," Oncotarget, vol. 8, no. 4, pp. 6955-6969, 2017.

[15] K. Ma, Y. He, H. Zhang et al., "DNA methylation-regulated miR-193a-3p dictates resistance of hepatocellular carcinoma to 5-fluorouracil via repression of SRSF2 expression," The Journal of Biological Chemistry, vol. 287, no. 8, pp. 56395649, 2012.

[16] J. Liz and M. Esteller, "IncRNAs and microRNAs with a role in cancer development," Biochimica et Biophysica Acta (BBA) Gene Regulatory Mechanisms, vol. 1859, no. 1, pp. 169-176, 2016.

[17] B. Yue, D. Cai, C. Liu, C. Fang, and D. Yan, "Linc00152 functions as a competing endogenous RNA to confer oxaliplatin resistance and holds prognostic values in colon cancer," Molecular Therapy, vol. 24, no. 12, pp. 2064-2077, 2016.

[18] W. Nie, H. J. Ge, X. Q. Yang et al., "LncRNA-UCA1 exerts oncogenic functions in non-small cell lung cancer by targeting 
miR-193a-3p," Cancer Letters, vol. 371, no. 1, pp. 99-106, 2016.

[19] GTEx Consortium, "The genotype-tissue expression (GTEx) project," Nature Genetics, vol. 45, no. 6, pp. 580-585, 2013.

[20] L. J. Carithers, K. Ardlie, M. Barcus et al., "A novel approach to high-quality postmortem tissue procurement: the GTEx project," Biopreservation and Biobanking, vol. 13, no. 5, pp. 311-319, 2015.

[21] C. P. Khoo, M. G. Roubelakis, J. B. Schrader et al., "miR-193a-3p interaction with HMGB1 downregulates human endothelial cell proliferation and migration," Scientific Reports, vol. 7, article 44137, 2017.

[22] S. Greco, A. Perfetti, P. Fasanaro et al., "Deregulated microRNAs in myotonic dystrophy type 2," PLoS One, vol. 7, no. 6, article e39732, 2012.

[23] S. Kuokkanen, B. Chen, L. Ojalvo, L. Benard, N. Santoro, and J. W. Pollard, "Genomic profiling of microRNAs and messenger RNAs reveals hormonal regulation in microRNA expression in human endometrium," Biology of Reproduction, vol. 82, no. 4, pp. 791-801, 2010.

[24] B. Mandriani, S. Castellana, C. Rinaldi et al., "Identification of p53-target genes in Danio rerio," Scientific Reports, vol. 6, article 32474, 2016.

[25] Y. Yi, J. Chen, C. Jiao et al., "Upregulated miR-193a-3p as an oncogene in esophageal squamous cell carcinoma regulating cellular proliferation, migration and apoptosis," Oncology Letters, vol. 12, no. 6, pp. 4779-4784, 2016.

[26] S. Uhlmann, H. Mannsperger, J. D. Zhang et al., "Global microRNA level regulation of EGFR-driven cell-cycle protein network in breast cancer," Molecular Systems Biology, vol. 8, p. 570, 2012.

[27] H. Liang, M. Liu, X. Yan et al., "miR-193a-3p functions as a tumor suppressor in lung cancer by down-regulating ERBB4," The Journal of Biological Chemistry, vol. 290, no. 2, pp. 926940, 2015.

[28] K. W. Tsai, C. M. Leung, Y. H. Lo et al., “Arm selection preference of microRNA-193a varies in breast cancer," Scientific Reports, vol. 6, article 28176, 2016.

[29] E. G. Seviour, V. Sehgal, D. Mishra et al., "Targeting KRasdependent tumour growth, circulating tumour cells and metastasis in vivo by clinically significant miR-193a-3p," Oncogene, vol. 36, no. 10, pp. 1339-1350, 2017.

[30] J. E. Kwon, B. Y. Kim, S. Y. Kwak, I. H. Bae, and Y. H. Han, "Ionizing radiation-inducible microRNA miR-193a-3p induces apoptosis by directly targeting Mcl-1," Apoptosis, vol. 18, no. 7, pp. 896-909, 2013.

[31] H. Nakano, Y. Yamada, T. Miyazawa, and T. Yoshida, "Gainof-function microRNA screens identify miR-193a regulating proliferation and apoptosis in epithelial ovarian cancer cells," International Journal of Oncology, vol. 42, no. 6, pp. 18751882, 2013.

[32] A. Salvi, I. Conde, E. Abeni et al., "Effects of miR-193a and sorafenib on hepatocellular carcinoma cells," Molecular Cancer, vol. 12, p. 162, 2013.

[33] H. Noh, S. Hong, Z. Dong, Z. K. Pan, Q. Jing, and S. Huang, "Impaired MicroRNA processing facilitates breast cancer cell invasion by upregulating urokinase-type plasminogen activator expression," Genes \& Cancer, vol. 2, no. 2, pp. 140-150, 2011.

[34] A. Salvi, B. Arici, A. Alghisi, S. Barlati, and G. De Petro, "RNA interference against urokinase in hepatocellular carcinoma xenografts in nude mice," Tumour Biology, vol. 28, no. 1, pp. 16-26, 2007.

[35] G. D. Petro, D. Tavian, A. Copeta, N. Portolani, S. M. Giulini, and S. Barlati, "Expression of urokinase-type plasminogen activator (u-PA), u-PA receptor, and tissue-type PA messenger RNAs in human hepatocellular carcinoma," Cancer Research, vol. 58, no. 10, pp. 2234-2239, 1998.

[36] J. A. Foekens, H. A. Peters, M. P. Look et al., "The urokinase system of plasminogen activation and prognosis in 2780 breast cancer patients," Cancer Research, vol. 60, no. 3, pp. 636-643, 2000.

[37] T. Yu, J. Li, M. Yan et al., "MicroRNA-193a-3p and $-5 p$ suppress the metastasis of human non-small-cell lung cancer by downregulating the ERBB4/PIK3R3/mTOR/S6K2 signaling pathway," Oncogene, vol. 34, no. 4, pp. 413-423, 2015.

[38] D. Jia, M. Yan, X. Wang et al., "Development of a highly metastatic model that reveals a crucial role of fibronectin in lung cancer cell migration and invasion," BMC Cancer, vol. 10, p. 364, 2010.

[39] W. Deng, M. Yan, T. Yu et al., "Quantitative proteomic analysis of the metastasis-inhibitory mechanism of miR-193a-3p in non-small cell lung cancer," Cellular Physiology and Biochemistry, vol. 35, no. 5, pp. 1677-1688, 2015.

[40] L. Lv, H. Deng, Y. Li et al., "The DNA methylation-regulated miR-193a-3p dictates the multi-chemoresistance of bladder cancer via repression of SRSF2/PLAU/HIC2 expression," Cell Death \& Disease, vol. 5, article e1402, 2014.

[41] H. Deng, L. Lv, Y. Li et al., "miR-193a-3p regulates the multidrug resistance of bladder cancer by targeting the LOXL4 gene and the oxidative stress pathway," Molecular Cancer, vol. 13, p. 234, 2014.

[42] Y. Li, H. Deng, L. Lv et al., "The miR-193a-3p-regulated ING5 gene activates the DNA damage response pathway and inhibits multi-chemoresistance in bladder cancer," Oncotarget, vol. 6, no. 12, pp. 10195-10206, 2015.

[43] L. Lv, Y. Li, H. Deng et al., "MiR-193a-3p promotes the multi-chemoresistance of bladder cancer by targeting the HOXC9 gene," Cancer Letters, vol. 357, no. 1, pp. 105113, 2015.

[44] H. Deng, L. Lv, Y. Li et al., "The miR-193a-3p regulated PSEN1 gene suppresses the multi-chemoresistance of bladder cancer," Biochimica et Biophysica Acta (BBA) - Molecular Basis of Disease, vol. 1852, no. 3, pp. 520-528, 2015.

[45] F. Meng, L. Qian, L. Lv et al., "miR-193a-3p regulation of chemoradiation resistance in oesophageal cancer cells via the PSEN1 gene," Gene, vol. 579, no. 2, pp. 139-145, 2016.

[46] X. Dai, X. Chen, Q. Chen et al., "MicroRNA-193a-3p reduces intestinal inflammation in response to microbiota via downregulation of colonic PepT1," The Journal of Biological Chemistry, vol. 290, no. 26, pp. 16099-16115, 2015.

[47] Y. Liu, F. Ren, Y. Luo, M. Rong, G. Chen, and Y. Dang, "Downregulation of MiR-193a-3p dictates deterioration of HCC: a clinical real-time qRT-PCR study," Medical Science Monitor, vol. 21, pp. 2352-2360, 2015.

[48] F. Ren, H. Ding, S. Huang et al., "Expression and clinicopathological significance of miR-193a-3p and its potential target astrocyte elevated gene-1 in non-small lung cancer tissues," Cancer Cell International, vol. 15, p. 80, 2015.

[49] S. Caramuta, S. Egyhazi, M. Rodolfo et al., "MicroRNA expression profiles associated with mutational status and 
survival in malignant melanoma," The Journal of Investigative Dermatology, vol. 130, no. 8, pp. 2062-2070, 2010.

[50] S. Gilad, E. Meiri, Y. Yogev et al., "Serum microRNAs are promising novel biomarkers," PLoS One, vol. 3, no. 9, article e3148, 2008.

[51] H. Dong, C. Wang, S. Lu et al., "A panel of four decreased serum microRNAs as a novel biomarker for early Parkinson's disease," Biomarkers, vol. 21, no. 2, pp. 129-137, 2016.

[52] H. Wei, Y. Yuan, S. Liu et al., "Detection of circulating miRNA levels in schizophrenia," The American Journal of Psychiatry, vol. 172, no. 11, pp. 1141-1147, 2015.

[53] F. L. Yong, C. W. Law, and C. W. Wang, "Potentiality of a triple microRNA classifier: miR-193a-3p, miR-23a and miR338-5p for early detection of colorectal cancer," BMC Cancer, vol. 13, p. 280, 2013.

[54] C. Wang, J. Hu, M. Lu et al., "A panel of five serum miRNAs as a potential diagnostic tool for early-stage renal cell carcinoma," Scientific Reports, vol. 5, p. 7610, 2015.

[55] C. Wang, M. Ding, M. Xia et al., "A five-miRNA panel identified from a multicentric case-control study serves as a novel diagnostic tool for ethnically diverse non-small-cell lung cancer patients," eBioMedicine, vol. 2, no. 10, pp. 1377-1385, 2015.

[56] C. Wu, C. Wang, X. Guan et al., "Diagnostic and prognostic implications of a serum miRNA panel in oesophageal squamous cell carcinoma," PLoS One, vol. 9, no. 3, article e92292, 2014.

[57] L. Li, J. G. Chen, X. Chen et al., "Serum miRNAs as predictive and preventive biomarker for pre-clinical hepatocellular carcinoma," Cancer Letters, vol. 373, no. 2, pp. 234-240, 2016.

[58] K. Zen and C. Y. Zhang, "Circulating microRNAs: a novel class of biomarkers to diagnose and monitor human cancers," Medicinal Research Reviews, vol. 32, no. 2, pp. 326-348, 2012.

[59] H. W. Liang, F. Gong, S. Y. Zhang, C. Y. Zhang, K. Zen, and $\mathrm{X}$. Chen, "The origin, function, and diagnostic potential of extracellular microRNAs in human body fluids," WIREs RNA, vol. 5, no. 2, pp. 285-300, 2014.

[60] H. Valadi, K. Ekstrom, A. Bossios, M. Sjöstrand, J. J. Lee, and J. O. Lötvall, "Exosome-mediated transfer of mRNAs and microRNAs is a novel mechanism of genetic exchange between cells," Nature Cell Biology, vol. 9, no. 6, pp. 654U672, 2007.

[61] H. J. Oh, Y. Shin, S. Chung, D. W. Hwang, and D. S. Lee, "Convective exosome-tracing microfluidics for analysis of cell-non-autonomous neurogenesis," Biomaterials, vol. 112, pp. 82-94, 2017.

[62] Y. Teng, Y. Ren, X. Hu et al., "MVP-mediated exosomal sorting of miR-193a promotes colon cancer progression," Nature Communications, vol. 8, article 14448, 2017.

[63] R. Rupaimoole and F. J. Slack, "MicroRNA therapeutics: towards a new era for the management of cancer and other diseases," Nature Reviews Drug Discovery, vol. 16, no. 3, pp. 203-222, 2017. 

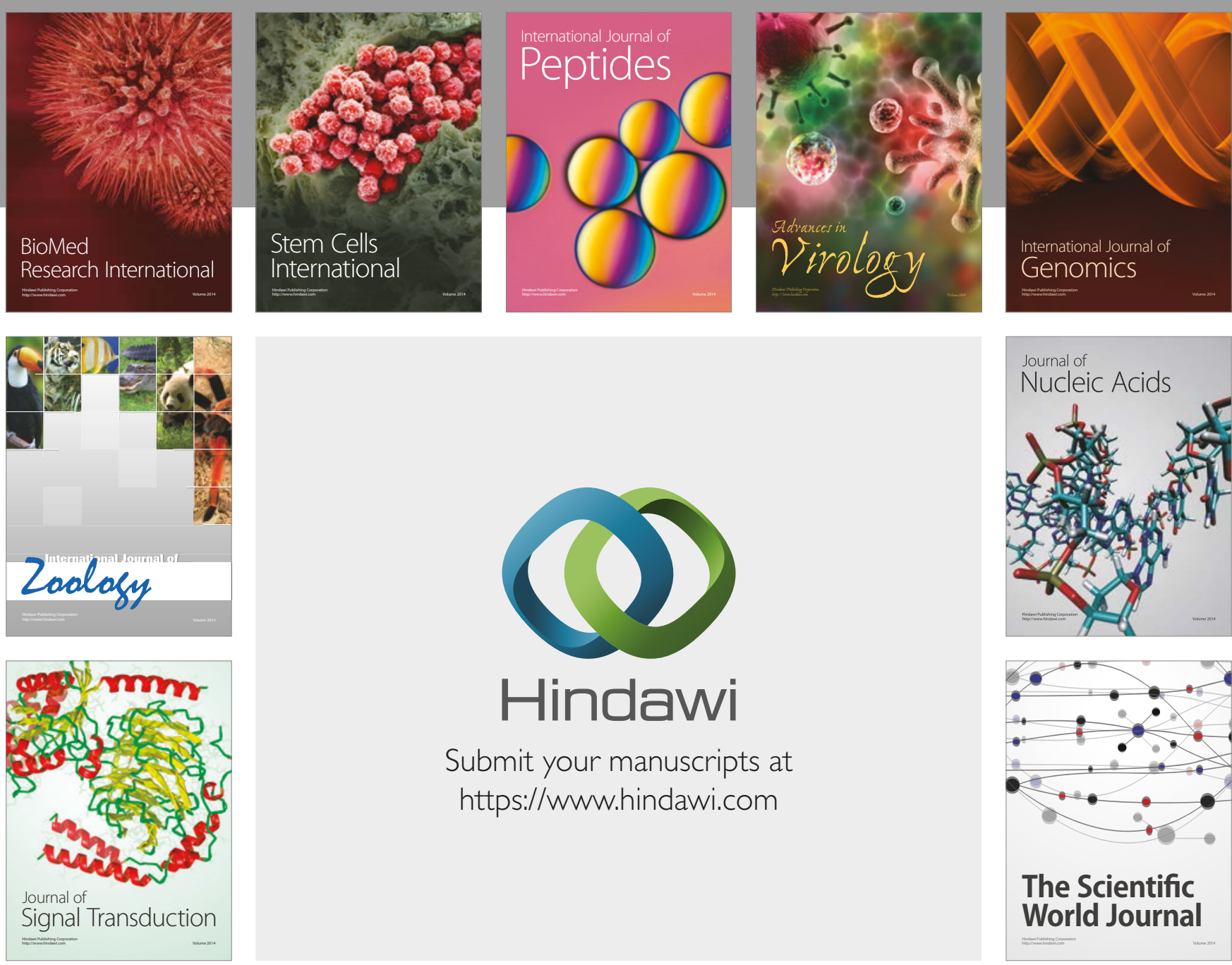

Submit your manuscripts at

https://www.hindawi.com
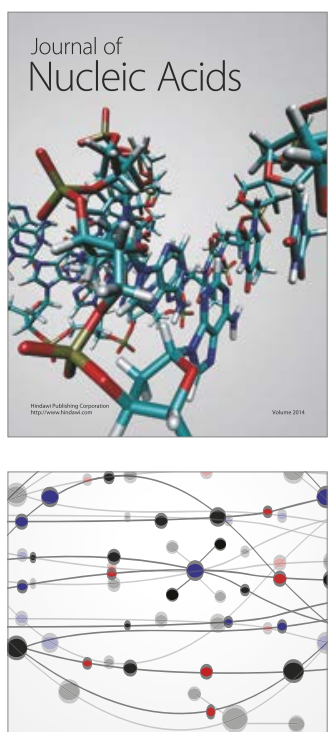

The Scientific World Journal

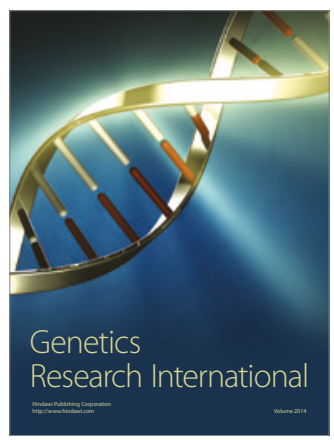

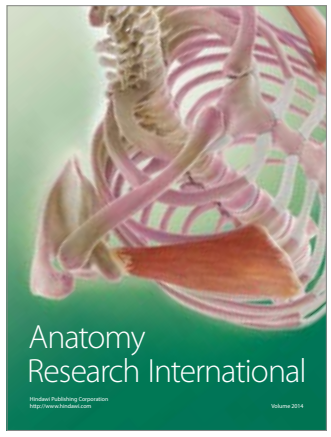

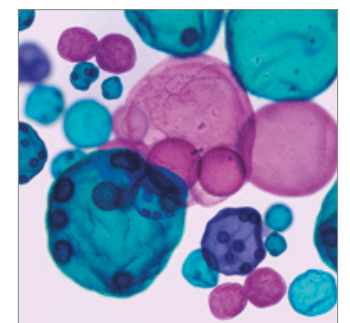

International Journal of Microbiology
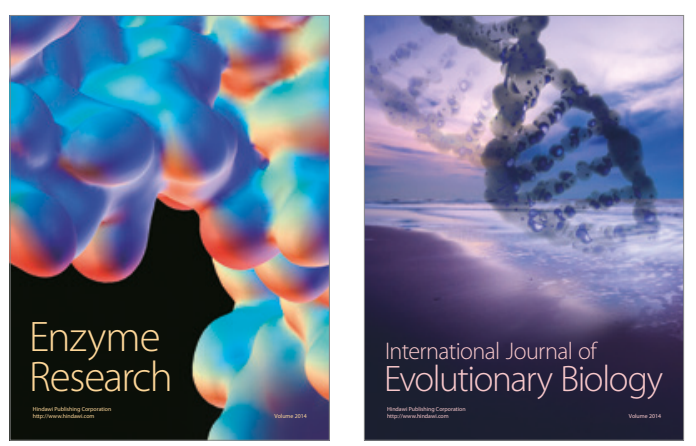
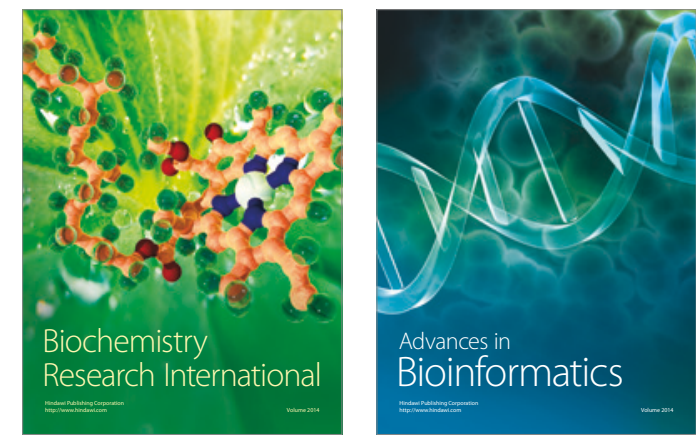

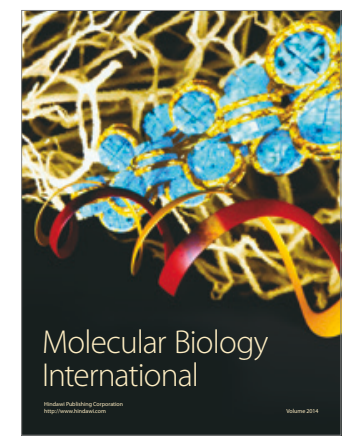

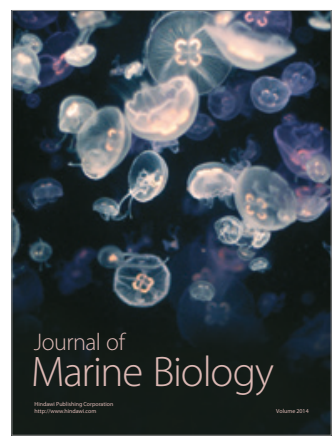

